\title{
Notes on Translational and Rotational Properties of Tensor Fields in Relativistic Quantum Mechanics
}

\author{
V. V. Dvoeglazov \\ UAF, Universidad de Zacatecas \\ Apartado Postal 636, Suc. 3 Cruces, Zacatecas 98068 Zac., México \\ Web: http://fisica.uaz.edu.mx/ valeri/ \\ E-mail: valeri@fisica.uaz.edu.mx
}

\begin{abstract}
Recently, several discussions on the possible observability of 4-vector fields have been published in literature. Furthermore, several authors recently claimed existence of the helicity $=0$ fundamental field. We re-examine the theory of antisymmetric tensor fields and 4-vector potentials. We study the massless limits. In fact, a theoretical motivation for this venture is the old papers of Ogievetskiı and Polubarinov, Hayashi, and Kalb and Ramond. Ogievetskiı and Polubarinov proposed the concept of the notoph, whose helicity properties are complementary to those of the photon. We analyze the quantum field theory with taking into account mass dimensions of the notoph and the photon. It appears to be possible to describe both photon and notoph degrees of freedom on the basis of the modified Bargmann-Wigner formalism for the symmetric second-rank spinor. Next, we proceed to derive equations for the symmetric tensor of the second rank on the basis of the Bargmann-Wigner formalism in a straightforward way. The symmetric multispinor of the fourth rank is used. Due to serious problems with the interpretation of the results obtained on using the standard procedure we generalize it and obtain the spin-2 relativistic equations, which are consistent with the general relativity. Thus, in fact we deduced the gravitational field equations from relativistic quantum mechanics. The relations of this theory with the scalar-tensor theories of gravitation and $f(R)$ are discussed. Particular attention has been paid to the correct definitions of the energy-momentum tensor and other Nöther currents in the electromagnetic theory, the relativistic theory of gravitation, the general relativity, and their generalizations. We estimate possible interactions, fermion-notoph, graviton-notoph, photon-notoph, and we conclude that they can probably be seen in experiments in the next few years.

PACS number: 03.65.Pm , 04.50.-h, 11.30.Cp
\end{abstract}

Keywords: Notoph; Graviton

\section{Introduction}

In this presentation we re-examine the theory of the 4-potential field, the antisymmetric tensor fields of the second ranks and the spin-2 fields coming from the modified Bargmann-Wigner formalism. In the series of the papers ${ }^{1-4}$ we tried to find connection between the theory of the quantized antisymmetric tensor (AST) field of the second rank (and that of the corresponding 4-vector field) with the $2(2 s+1)$ Weinberg-Tucker-Hammer formalism. ${ }^{5,6}$ Several previously published works, Refs., ${ }^{7-11}$ introduced the concept of the notoph (the Kalb-Ramond field) which is constructed on the basis of the antisymmetric tensor "potentials". It represents itself the non-trivial spin-0 field. The well-known textbooks ${ }^{12-14}$ did not discuss the problems, whether the massless quantized AST "potential" and the quantized 4-vector field are transverse or longitudinal fields (in the sense if the helicity $h= \pm 1$ or $h=0)$ ? can the electromagnetic potential be a 4 -vector in a quantized theory ? contradictions with the Weinberg theorem "that no sym- metric tensor field of rank $s$ can be constructed from the creation and annihilation operators of massless particles of spin $s$ "? how should the massless limit be taken? and many other fundamental problems of the physics of bosons. However, one can advise Refs. ${ }^{5,15}$

First of all, after a referee of "Found. Phys." and "Int. J. Mod. Phys." we note that 1) "...In natural units $(c=\hbar=1) \ldots$ a lagrangian density, since the action is dimensionless, has dimension of [energy] ${ }^{4}$; 2) One can always renormalize the lagrangian density and "one can obtain the same equations of motion... by substituting $L \rightarrow\left(1 / M^{N}\right) L$, where $M$ is an arbitrary energy scale", cf. Ref. $\left.{ }^{3} 3\right)$ the right physical dimension of the field strength tensor $F^{\mu \nu}$ is [energy] ${ }^{2}$; "the transformation $F^{\mu \nu} \rightarrow(1 / 2 m) F^{\mu \nu}$ [which was regarded in Ref. ${ }^{16,17}$ ] ... requires a more detailed study ... [because] the transformation above changes its physical dimension: it is not a simple normalization transformation". Furthermore, in the first papers on the notoph ${ }^{8-10}$ the authors used the normalization of the 4 -vector $F^{\mu}$ field (which is related to a third-rank antisymmetric field tensor) to 
[energy $]^{2}$ and, hence, the antisymmetric tensor "potentials" $A^{\mu \nu}$, to [energy] $]^{1}$. We try to discuss these problems on the basis of the generalized BargmannWigner formalism. ${ }^{18}$ The Proca and Maxwell formalisms are generalized too, see, e. g., Ref. ${ }^{4}$ In the next Sections we consider the spin-2 equations. A field of the rest mass $m$ and the spin $s \geq \frac{1}{2}$ is represented by a completely symmetric multispinor of rank $2 s$. The particular cases $s=1$ and $s=\frac{3}{2}$ have been considered in the textbooks, e. g., Ref. ${ }^{19}$ The spin-2 case can also be of some interest. Nevertheless, questions of the redundant components of the higher-spin relativistic equations are not yet understood in detail. ${ }^{20}$ In the last Sections (in the previous papers of us, as well) we discuss the questions of quantization, interactions and relations between various higher-spin theories.

\section{4-potentials and Antisymmetric Tensor Field. Normalization.}

The spin-0 and spin-1 field particles can be constructed by taking the direct product of 4spinors. ${ }^{18,19}$ Let us firstly repeat the BargmannWigner procedure for bosons of spin 0 and 1 . The set of basic equations for $s=0$ and $s=1$ are written, e.g., in Ref. ${ }^{19}$

$$
\begin{aligned}
& {\left[i \gamma^{\mu} \partial_{\mu}-m\right]_{\alpha \beta} \Psi_{\beta \gamma}(x)=0,} \\
& {\left[i \gamma^{\mu} \partial_{\mu}-m\right]_{\gamma \beta} \Psi_{\alpha \beta}(x)=0 .}
\end{aligned}
$$

We expand the $4 \times 4$ matrix field function into the antisymmetric and symmetric parts in the standard way

$$
\begin{aligned}
\Psi_{[\alpha \beta]} & =R_{\alpha \beta} \phi+\gamma_{\alpha \delta}^{5} R_{\delta \beta} \widetilde{\phi}+\gamma_{\alpha \delta}^{5} \gamma_{\delta \tau}^{\mu} R_{\tau \beta} \widetilde{A}_{\mu} \\
\Psi_{\{\alpha \beta\}} & =\gamma_{\alpha \delta}^{\mu} R_{\delta \beta} A_{\mu}+\sigma_{\alpha \delta}^{\mu \nu} R_{\delta \beta} F_{\mu \nu}
\end{aligned}
$$

where $R=C P$ has the properties (which are necessary to make expansions $(3,4)$ to be possible in such a form)

$$
\begin{aligned}
& R^{T}=-R, R^{\dagger}=R=R^{-1} \\
& R^{-1} \gamma^{5} R=\left(\gamma^{5}\right)^{T} \\
& R^{-1} \gamma^{\mu} R=-\left(\gamma^{\mu}\right)^{T} \\
& R^{-1} \sigma^{\mu \nu} R=-\left(\sigma^{\mu \nu}\right)^{T}
\end{aligned}
$$

The explicit form of this matrix can be chosen:

$$
R=\left(\begin{array}{cc}
i \Theta & 0 \\
0 & -i \Theta
\end{array}\right), \Theta=-i \sigma_{2}=\left(\begin{array}{cc}
0 & -1 \\
1 & 0
\end{array}\right),
$$

provided that $\gamma^{\mu}$ matrices are in the Weyl representation. The equations $(1,2)$ lead to the Kemmer set of the $s=0$ equations:

$$
\begin{aligned}
m \phi & =0, \\
m \widetilde{\phi} & =-i \partial_{\mu} \widetilde{A}^{\mu}, \\
m \widetilde{A^{\mu}} & =-i \partial^{\mu} \widetilde{\phi},
\end{aligned}
$$

and to the Proca-Duffin-Kemmer set of the equations for the $s=1$ case: $^{\mathrm{b}}$

$$
\begin{aligned}
& \partial_{\alpha} F^{\alpha \mu}+\frac{m}{2} A^{\mu}=0, \\
& 2 m F^{\mu \nu}=\partial^{\mu} A^{\nu}-\partial^{\nu} A^{\mu},
\end{aligned}
$$

In the meantime, in the textbooks, the latter set is usually written as (e.g., p. 135 of Ref. ${ }^{14}$ )

$$
\begin{aligned}
& \partial_{\alpha} F^{\alpha \mu}+m^{2} A^{\mu}=0, \\
& F^{\mu \nu}=\partial^{\mu} A^{\nu}-\partial^{\nu} A^{\mu},
\end{aligned}
$$

The set $(17,18)$ is obtained from $(15,16)$ after the normalization change $A_{\mu} \rightarrow 2 m A_{\mu}$, or $F_{\mu \nu} \rightarrow \frac{1}{2 m} F_{\mu \nu}$. Of course, one can investigate other sets of equations with different normalizations of the $F_{\mu \nu}$ and $A_{\mu}$ fields. Are all these sets of equations equivalent? As we can see, to answer this question is not trivial. At the moment, we mention that the massless limit can and must be taken in the end of calculations only, i. e., for physical quantities.

In order to be able to answer the question about the behaviour of eigenvalues of the spin operator $\mathbf{J}^{i}=\frac{1}{2} \epsilon^{i j k} J^{j k}$ in the massless limit one should know the behaviour of the fields $F_{\mu \nu}$ and/or $A_{\mu}$ in the massless limit. We want to analyze the first set

\footnotetext{
${ }^{\mathrm{b}}$ We could use another symmetric matrix $\gamma^{5} \sigma^{\mu \nu} R$ in the expansion of the symmetric spinor of the second rank. ${ }^{17}$ In this case the equations are

$$
\begin{aligned}
& i \partial_{\alpha} \widetilde{F}^{\alpha \mu}+\frac{m}{2} B^{\mu}=0, \\
& 2 i m \widetilde{F}^{\mu \nu}=\partial^{\mu} B^{\nu}-\partial^{\nu} B^{\mu},
\end{aligned}
$$

in which the dual tensor $\widetilde{F}^{\mu \nu}=\frac{1}{2} \epsilon^{\mu \nu \rho \sigma} F_{\rho \sigma}$ presents, because we used that in the Weyl representation $\gamma^{5} \sigma^{\mu \nu}=\frac{i}{2} \epsilon^{\mu \nu \rho \sigma} \sigma_{\rho \sigma}$; $B^{\mu}$ is the corresponding vector potential. The equation for the antisymmetric tensor field (which can be obtained from this set) does not change its form but we see some "renormalization" of the field functions. In general, it is permitted to choose various relative factors in the expansions of the symmetric wave function (4). It is also permitted to consider the matrix term of the form $\gamma^{5} \sigma^{\mu \nu}$. We have additional factors in equations connecting physical fields and their potentials. They can be absorbed by redefinitions of the potentials/fields. The above shows that the dual tensor of the second rank can also be epxanded in potentials. See below.
} 
$(15,16)$. If one chooses the following definitions ( $p$. 209 of Ref. ${ }^{15}$ )

$$
\begin{aligned}
& \epsilon^{\mu}(\mathbf{0},+1)=-\frac{1}{\sqrt{2}}\left(\begin{array}{l}
0 \\
1 \\
i \\
0
\end{array}\right), \epsilon^{\mu}(\mathbf{0}, 0)=\left(\begin{array}{l}
0 \\
0 \\
0 \\
1
\end{array}\right), \\
& \epsilon^{\mu}(\mathbf{0},-1)=\frac{1}{\sqrt{2}}\left(\begin{array}{c}
0 \\
1 \\
-i \\
0
\end{array}\right),
\end{aligned}
$$

and $\left(\widehat{p}_{i}=p_{i} /|\mathbf{p}|, \gamma=E_{p} / m\right)$, p. 68 of Ref., ${ }^{15}$

$$
\begin{aligned}
& \epsilon^{\mu}(\mathbf{p}, \sigma)=L_{\nu}^{\mu}(\mathbf{p}) \epsilon^{\nu}(\mathbf{0}, \sigma) \\
& L_{0}^{0}(\mathbf{p})=\gamma, L_{0}^{i}(\mathbf{p})=L_{i}^{0}(\mathbf{p})=\widehat{p}_{i} \sqrt{\gamma^{2}-1} \\
& L_{k}^{i}(\mathbf{p})=\delta_{i k}+(\gamma-1) \widehat{p}_{i} \widehat{p}_{k}
\end{aligned}
$$

for the 4-vector potential field, ${ }^{\mathrm{c}}$ p. 129 of Ref. ${ }^{14}$ functions in the momentum representation:

$$
\begin{aligned}
& u^{\mu}(\mathbf{p},+1)=-\frac{N}{\sqrt{2} m}\left(\begin{array}{c}
p_{r} \\
m+\frac{p_{1} p_{r}}{E_{p}+m} \\
i m+\frac{p_{2} p_{r}}{E_{p}+m} \\
\frac{p_{3} p_{r}}{E_{p}+m}
\end{array}\right), \\
& u^{\mu}(\mathbf{p},-1)=\frac{N}{\sqrt{2} m}\left(\begin{array}{c}
p_{l} \\
m+\frac{p_{1} p_{l}}{E_{p}+m} \\
-i m+\frac{p_{2} p_{l}}{E_{p}+m} \\
\frac{p_{3} p_{l}}{E_{p}+m}
\end{array}\right),
\end{aligned}
$$

$$
u^{\mu}(\mathbf{p}, 0)=\frac{N}{m}\left(\begin{array}{c}
p_{3} \\
\frac{p_{1} p_{3}}{E_{p}+m} \\
\frac{p_{2} p_{3}}{E_{p}+m} \\
m+\frac{p_{3}^{2}}{E_{p}+m}
\end{array}\right)
$$

$\left(N=m\right.$ and $\left.p_{r, l}=p_{1} \pm i p_{2}\right)$ which do not diverge in the massless limit. Two of the massless functions (24) (with $\sigma= \pm 1$ ) are equal to zero when a particle, described by this field, is moving along the third axis $\left(p_{1}=p_{2}=0, p_{3} \neq 0\right)$. The third one $(\sigma=0)$ is

$$
\left.u^{\mu}\left(p_{3}, 0\right)\right|_{m \rightarrow 0}=\left(\begin{array}{c}
p_{3} \\
0 \\
0 \\
\frac{p_{3}^{2}}{E_{p}}
\end{array}\right) \equiv\left(\begin{array}{c}
E_{p} \\
0 \\
0 \\
E_{p}
\end{array}\right)
$$

and at the rest $\left(E_{p}=p_{3} \rightarrow 0\right)$ also vanishes. Thus, such a field operator describes the "longitudinal photons" what is in the complete accordance with the Weinberg theorem $B-A=h$ for massless particles (we use the $D(1 / 2,1 / 2)$ representation). Thus, the change of the normalization can lead to the "change" of physical content described by the classical field. In the quantum case one should somehow fix the form of commutation relations by some physical principles. They may be fixed by requirements of the dimensionless of the action in the natural unit system (apart from the requirements of the translational and rotational invariancies; the accustomed behaviour of the Feynman-Dyson propagator), etc.

Furthermore, it is easy to find the properties of the physical fields $F^{\mu \nu}$ (defined as in $(15,16)$, for instance) in the massless zero-momentum limit. It is straightforward to find $\mathbf{B}^{(+)}(\mathbf{p}, \sigma)=\frac{i}{2 m} \mathbf{p} \times \mathbf{u}(\mathbf{p}, \sigma)$, $\mathbf{E}^{(+)}(\mathbf{p}, \sigma)=\frac{i}{2 m} p_{0} \mathbf{u}(\mathbf{p}, \sigma)-\frac{i}{2 m} \mathbf{p} u^{0}(\mathbf{p}, \sigma)$ and the corresponding negative-energy strengths for the field 
operator (in general, complex-valued)

$$
\begin{aligned}
& F^{\mu \nu}=\sum_{\sigma=0, \pm 1} \int \frac{d^{3} \mathbf{p}}{(2 \pi)^{3} 2 E_{p}}\left[F_{(+)}^{\mu \nu}(\mathbf{p}, \sigma) a(\mathbf{p}, \sigma) e^{-i p x}\right. \\
& \left.+F_{(-)}^{\mu \nu}(\mathbf{p}, \sigma) b^{\dagger}(\mathbf{p}, \sigma) e^{+i p x}\right]
\end{aligned}
$$

see Refs. ${ }^{16,22}$

For the sake of completeness let us present the vector corresponding to the "time-like" polarization:

$$
\begin{aligned}
& u^{\mu}\left(\mathbf{p}, 0_{t}\right)=\frac{N}{m}\left(\begin{array}{c}
E_{p} \\
p_{1} \\
p_{2} \\
p_{3}
\end{array}\right), \\
& \mathbf{B}^{( \pm)}\left(\mathbf{p}, 0_{t}\right)=\mathbf{0}, \mathbf{E}^{( \pm)}\left(\mathbf{p}, 0_{t}\right)=\mathbf{0}
\end{aligned}
$$

The polarization vector $u^{\mu}\left(\mathbf{p}, 0_{t}\right)$ has good behaviour in $m \rightarrow 0, N=m$ (and also in the subsequent limit $\mathbf{p} \rightarrow \mathbf{0}$ ) and it may correspond to some field (particle). As one can see the field operator may describe a situation when a particle and an antiparticle have opposite intrinsic parities, if it was composed of the state of longitudinal polazrization (e.g., as the "positive-energy" solution) and that of time-like polarization (e.g., as the "negative-energy" solution). Furthermore, in the case of the normalization of potentials to the mass $N=m$ the physical fields $\mathbf{B}$ and E, which correspond to the "time-like" polarization, are equal to zero identically. The longitudinal fields (strengths) are equal to zero in this limit only when one chooses the frame with $p_{3}=|\mathbf{p}|$, cf. with the light front formulation, Ref. $^{2}$ In the case $N=1$ and $(15,16)$ the fields $\mathbf{B}^{ \pm}\left(\mathbf{p}, 0_{t}\right)$ and $\mathbf{E}^{ \pm}\left(\mathbf{p}, 0_{t}\right)$ would be undefined.

\section{Lagrangian, Energy-Momentum Tensor and Angular Momentum.}

We begin with the Lagrangian, including, in general, mass term: ${ }^{\mathrm{a}}$

$$
\begin{aligned}
\mathcal{L} & =\frac{1}{4}\left(\partial_{\mu} A_{\nu \alpha}\right)\left(\partial^{\mu} A^{\nu \alpha}\right)-\frac{1}{2}\left(\partial_{\mu} A^{\mu \alpha}\right)\left(\partial^{\nu} A_{\nu \alpha}\right)- \\
& -\frac{1}{2}\left(\partial_{\mu} A_{\nu \alpha}\right)\left(\partial^{\nu} A^{\mu \alpha}\right)+\frac{1}{4} m^{2} A_{\mu \nu} A^{\mu \nu}
\end{aligned}
$$

The Lagrangian leads to the equation of motion in the following form (provided that the appropriate antisymmetrization procedure has been taken into account):

$$
\frac{1}{2}\left(\square+m^{2}\right) A_{\mu \nu}+\left(\partial_{\mu} A_{\alpha \nu}^{, \alpha}-\partial_{\nu} A_{\alpha \mu}^{, \alpha}\right)=0,
$$

where $\square=-\partial_{\alpha} \partial^{\alpha}$, cf. with the set of equations $(15,16)$. It is this equation for antisymmetric-tensorfield components that follows from the Proca-DuffinKemmer consideration provided that $m \neq 0$ and in the final expression one takes into account the KleinGordon equation $\left(\square-m^{2}\right) A_{\mu \nu}=0$. The latter expresses relativistic dispersion relations $E^{2}-\mathbf{p}^{2}=m^{2}$.

Following the variation procedure one can obtain the energy-momentum tensor:

$$
\begin{aligned}
\Theta^{\lambda \beta} & =\frac{1}{2}\left[\left(\partial^{\lambda} A_{\mu \alpha}\right)\left(\partial^{\beta} A^{\mu \alpha}\right)-2\left(\partial_{\mu} A^{\mu \alpha}\right)\left(\partial^{\beta} A_{\alpha}^{\lambda}\right)-\right. \\
& \left.-2\left(\partial^{\mu} A^{\lambda \alpha}\right)\left(\partial^{\beta} A_{\mu \alpha}\right)\right]-\mathcal{L} g^{\lambda \beta} .
\end{aligned}
$$

One can also obtain that for rotations $x^{\mu^{\prime}}=x^{\mu}+$ $\omega^{\mu \nu} x_{\nu}$ the corresponding variation of the wave func-

a Here we use the notation $A_{\mu \nu}$ for the AST due to different "mass dimension" of the fields. The massless $(m=0)$ Lagrangian is connected with the Lagrangians used in the conformal field theories by adding the total derivative:

$$
\mathcal{L}_{C F T}=\mathcal{L}+\frac{1}{2} \partial_{\mu}\left(A_{\nu \alpha} \partial^{\nu} A^{\mu \alpha}-A^{\mu \alpha} \partial^{\nu} A_{\nu \alpha}\right) .
$$

The Kalb-Ramond gauge-invariant form (with respect to "gauge" transformations $\left.A_{\mu \nu} \rightarrow A_{\mu \nu}+\partial_{\nu} \Lambda_{\mu}-\partial_{\mu} \Lambda_{\nu}\right)$, Refs. ${ }^{8-10}$ is obtained only if one uses the Fermi procedure mutatis mutandis by removing the additional "phase" field $\lambda\left(\partial_{\mu} A^{\mu \nu}\right)^{2}$ from the Lagrangian. This has certain analogy with the QED, where the question, whether the Lagrangian is gauge-invariant or not, is solved depending on the presence of the term $\lambda\left(\partial_{\mu} A^{\mu}\right)^{2}$. For details see Refs. ${ }^{9,10}$

In general it is possible to introduce various forms of the mass term and forms of corresponding normalization of the field. But, the dimensionless of the action $\mathcal{S}$ imposes some restrictions. We know that $A^{\mu \nu}$ (in order to be able to describe long-range forces) should have the dimension [energy $]^{2}$ in the natural unit system. In order to take this into account one should divide the Lagrangian (33) by $\mathrm{m}^{2}$; calculate corresponding dynamical invariants, other observable quantities; and only then study $m \rightarrow 0$ limit. 
tion is found from the formula:

$$
\delta A^{\alpha \beta}=\frac{1}{2} \omega^{\kappa \tau} \mathcal{T}_{\kappa \tau}^{\alpha \beta, \mu \nu} A_{\mu \nu} .
$$

The generators of infinitesimal transformations are then defined as

$$
\begin{gathered}
\mathcal{T}_{\kappa \tau}^{\alpha \beta, \mu \nu}=\frac{1}{2} g^{\alpha \mu}\left(\delta_{\kappa}^{\beta} \delta_{\tau}^{\nu}-\delta_{\tau}^{\beta} \delta_{\kappa}^{\nu}\right)+\frac{1}{2} g^{\beta \mu}\left(\delta_{\kappa}^{\nu} \delta_{\tau}^{\alpha}-\delta_{\tau}^{\nu} \delta_{\kappa}^{\alpha}\right) \\
+\frac{1}{2} g^{\alpha \nu}\left(\delta_{\kappa}^{\mu} \delta_{\tau}^{\beta}-\delta_{\tau}^{\mu} \delta_{\kappa}^{\beta}\right)+\frac{1}{2} g^{\beta \nu}\left(\delta_{\kappa}^{\alpha} \delta_{\tau}^{\mu}-\delta_{\tau}^{\alpha} \delta_{\kappa}^{\mu}(\beta .7)\right.
\end{gathered}
$$

It is $\mathcal{T}_{\kappa \tau}^{\alpha \beta, \mu \nu}$, the generators of infinitesimal transformations, that enter in the formula for the relativistic spin tensor:

$$
J_{\kappa \tau}=\int d^{3} \mathbf{x}\left[\frac{\partial \mathcal{L}}{\partial\left(\partial A^{\alpha \beta} / \partial t\right)} \mathcal{T}_{\kappa \tau}^{\alpha \beta, \mu \nu} A_{\mu \nu}\right]
$$

As a result one obtains:

$$
\begin{aligned}
J_{\kappa \tau} & =\int d^{3} \mathbf{x}\left[\left(\partial_{\mu} A^{\mu \nu}\right)\left(g_{0 \kappa} A_{\nu \tau}-g_{0 \tau} A_{\nu \kappa}\right)-\right. \\
& -\left(\partial_{\mu} A^{\mu}{ }_{\kappa}\right) A_{0 \tau}+\left(\partial_{\mu} A^{\mu}{ }_{\tau}\right) A_{0 \kappa}+ \\
& +A_{\kappa}^{\mu}\left(\partial_{0} A_{\tau \mu}+\partial_{\mu} A_{0 \tau}+\partial_{\tau} A_{\mu 0}\right) \\
& \left.-A_{\tau}^{\mu}\left(\partial_{0} A_{\kappa \mu}+\partial_{\mu} A_{0 \kappa}+\partial_{\kappa} A_{\mu 0}\right)\right]
\end{aligned}
$$

If one agrees that the orbital part of the angular momentum

$$
L_{\kappa \tau}=x_{\kappa} \Theta_{0 \tau}-x_{\tau} \Theta_{0 \kappa}
$$

with $\Theta_{\tau \lambda}$ being the energy-momentum tensor, does not contribute to the Pauli-Lubanski operator when acting on the one-particle free states (as in the Dirac $s=1 / 2$ case), then the Pauli-Lubanski 4-vector is constructed as follows, Eq. (2-21) of Ref. ${ }^{14}$ :

$$
W_{\mu}=-\frac{1}{2} \epsilon_{\mu \kappa \tau \nu} J^{\kappa \tau} P^{\nu}
$$

with $J^{\kappa \tau}$ defined by Eqs. $(38,39)$. The 4-momentum operator $P^{\nu}$ can be replaced by its eigenvalue when acting on the plane-wave eigenstates.

Furthermore, one should choose space-like normalized vector $n^{\mu} n_{\mu}=-1$, for example $n_{0}=0$, $\mathbf{n}=\widehat{\mathbf{p}}=\mathbf{p} /|\mathbf{p}|{ }^{\mathrm{b}} \quad$ After lengthy calculations in a

\footnotetext{
${ }^{\mathrm{b}}$ One should remember that the helicity operator is usually connected with the Pauli-Lubanski vector in the following manner $(\mathbf{J} \cdot \widehat{\mathbf{p}})=(\mathbf{W} \cdot \widehat{\mathbf{p}}) / E_{p}$, see Ref. ${ }^{24}$ The choice of Ref., ${ }^{14}$ p. $147, n^{\mu}=\left(t^{\mu}-p^{\mu} \frac{p \cdot t}{m^{2}}\right) \frac{m}{|\mathbf{p}|}$, with $t^{\mu} \equiv(1,0,0,0)$ being a time-like vector, is also possible but it leads to some oscurities in the procedure of taking the massless limit.
}

spirit of pp. 58, 147 of Ref., ${ }^{14}$ one can find the explicit form of the relativistic spin:

$$
\begin{gathered}
\left(W_{\mu} \cdot n^{\mu}\right)=-(\mathbf{W} \cdot \mathbf{n})=-\frac{1}{2} \epsilon^{i j k} n^{k} J^{i j} p^{0} \\
\mathbf{J}^{k}=\frac{1}{2} \epsilon^{i j k} J^{i j}=\epsilon^{i j k} \int d^{3} \mathbf{x}\left[A^{0 i}\left(\partial_{\mu} A^{\mu j}\right)+\right. \\
\left.+A_{\mu}{ }^{j}\left(\partial^{0} A^{\mu i}+\partial^{\mu} A^{i 0}+\partial^{i} A^{0 \mu}\right)\right] .
\end{gathered}
$$

Now it becomes obvious that the application of the generalized Lorentz conditions (which are the quantum versions of free-space dual Maxwell's equations) leads in such a formulation to the absence of electromagnetism in a conventional sense. The resulting Kalb-Ramond field is longitudinal (helicity $h=0$ ). All the components of the angular momentum tensor for this case are identically equated to zero.

According to $[8$, Eqs. $(9,10)]$ we proceed in the construction of the "potentials" for the notoph :

$\tilde{F}_{\mu \nu}(\mathbf{p}) \sim A_{\mu \nu}(\mathbf{p})=N\left[\epsilon_{\mu}^{(1)}(\mathbf{p}) \epsilon_{\nu}^{(2)}(\mathbf{p})-\epsilon_{\nu}^{(1)}(\mathbf{p}) \epsilon_{\mu}^{(2)}(\mathbf{p})\right]$

On using explicit forms for the polarization vectors in the momentum space one obtains

$$
A^{\mu \nu}=\frac{i N^{2}}{m}\left(\begin{array}{cccc}
0 & -p_{2} & p_{1} & 0 \\
p_{2} & 0 & m+\frac{p_{r} p_{l}}{p_{0}+m} & \frac{p_{2} p_{3}}{p_{0}+m} \\
-p_{1}-m-\frac{p_{r} p_{l}}{p_{0}+m} & 0 & -\frac{p_{1} p_{3}}{p_{0}+m} \\
0 & -\frac{p_{2} p_{3}}{p_{0}+m} & \frac{p_{1} p_{3}}{p_{0}+m} & 0
\end{array}\right)
$$

i.e., it coincides with the longitudinal components of the antisymmetric tensor obtained in Refs. [1, Eqs.(2.14,2.17)] and [16, Eqs.(17b,18b)] within the normalization and different forms of the spin basis. The longitudinal states reduce to zero in the massless case under appropriate choice of the normalization and only if a $s=1$ particle moves along with the third axis $O Z$.

Finally, we agree with the previous authors, e. g., Ref. $^{23}$, see Eq. (4) therein, about the gauge noninvariance of the separation of the angular momentum of the electromagnetic field into the "orbital" and "spin" part (43). We proved again that for the antisymmetric tensor field $\mathbf{J} \sim \int d^{3} \mathbf{x}(\mathbf{E} \times \mathbf{A})$. So, what people actually did (when spoken about the Ogievetskiı-Polubarinov-Kalb-Ramond field) is: When $N=m$ they considered the gauge part of the 4-vector field functions. Then, they equated $\mathbf{A}$ of the transverse modes on choosing $p_{r}=p_{l}=0$ in 
the massless limit (see formulas (26)). ${ }^{\mathrm{c}}$ Under this choice the $\mathbf{E}(\mathbf{p}, 0)$ and $\mathbf{B}(\mathbf{p}, 0)$ are equal to zero in massless limit. But, the gauge part of $u^{\mu}(\mathbf{p}, 0)$ is not. The spin angular momentum can still be zero.

\section{The Relations with the $2(2 s+1)$ Formalism. Photon-Notoph Equations.}

For the spin 1 one can start from

$$
\left[\gamma_{\alpha \beta} p^{\alpha} p^{\beta}-A p^{\alpha} p_{\alpha}+B m^{2}\right] \Psi=0,
$$

where $p_{\mu}=-i \partial_{\mu}$ and $\gamma_{\alpha \beta}$ are the Barut-MuzinichWilliams covariantly-defined $6 \times 6$ matrices. One can consider four cases:

- $\Psi^{(I)}=\left(\begin{array}{l}\mathbf{E}+i \mathbf{B} \\ \mathbf{E}-i \mathbf{B}\end{array}\right), P=-1$, where $\mathbf{E}_{i}$ and $\mathbf{B}_{i}$ are the components of the tensor.

- $\Psi^{(I I)}=\left(\begin{array}{l}\mathbf{B}-i \mathbf{E} \\ \mathbf{B}+i \mathbf{E}\end{array}\right), P=+1$, where $\mathbf{E}_{i}, \mathbf{B}_{i}$ are the components of the tensor.

- $\Psi^{(I I I)}=\Psi^{(I)}$, but $\mathbf{E}_{i}$ and $\mathbf{B}_{i}$ are the corresponding vector and axial-vector components of the dual tensor $\tilde{F}_{\mu \nu}$.

- $\Psi^{(I V)}=\Psi^{(I I)}$, where $\mathbf{E}_{i}$ and $\mathbf{B}_{i}$ are the components of the dual tensor $\tilde{F}_{\mu \nu}$.

The mappings of the Weinberg-Tucker-Hammer (WTH) equations are:

$$
\begin{aligned}
& \partial_{\alpha} \partial^{\mu} F_{\mu \beta}^{(I)}-\partial_{\beta} \partial^{\mu} F_{\mu \alpha}^{(I)} \\
+ & \frac{A-1}{2} \partial^{\mu} \partial_{\mu} F_{\alpha \beta}^{(I)}+\frac{B}{2} m^{2} F_{\alpha \beta}^{(I)}=0, \\
& \partial_{\alpha} \partial^{\mu} F_{\mu \beta}^{(I I)}-\partial_{\beta} \partial^{\mu} F_{\mu \alpha}^{(I I)} \\
- & \frac{A+1}{2} \partial^{\mu} \partial_{\mu} F_{\alpha \beta}^{(I I)}-\frac{B}{2} m^{2} F_{\alpha \beta}^{(I I)}=0, \\
& \partial_{\alpha} \partial^{\mu} \tilde{F}_{\mu \beta}^{(I I I)}-\partial_{\beta} \partial^{\mu} \tilde{F}_{\mu \alpha}^{(I I)} \\
- & \frac{A+1}{2} \partial^{\mu} \partial_{\mu} \tilde{F}_{\alpha \beta}^{(I I I)}-\frac{B}{2} m^{2} \tilde{F}_{\alpha \beta}^{(I I I)}=0, \\
& \partial_{\alpha} \partial^{\mu} \tilde{F}_{\mu \beta}^{(I V)}-\partial_{\beta} \partial^{\mu} \tilde{F}_{\mu \alpha}^{(I V)} \\
+ & \frac{A-1}{2} \partial^{\mu} \partial_{\mu} \tilde{F}_{\alpha \beta}^{(I V)}+\frac{B}{2} m^{2} \tilde{F}_{\alpha \beta}^{(I V)}=0,
\end{aligned}
$$

where the superindices $(I)-(I V)$ refer to different forms of the WTH field functions, which are composed from $\mathbf{E}$ and $\mathbf{B}$, polar and axial 3-vectors. In the

$\overline{{ }^{c}}$ The reader, of course, can consider this procedure as the usual gauge transformation, $A^{\mu} \rightarrow A^{\mu}+\partial^{\mu} \chi$.
Tucker-Hammer case $(A=1, B=2)$ we can recover the Proca theory from (47):

$$
\partial_{\alpha} \partial^{\mu} F_{\mu \beta}-\partial_{\beta} \partial^{\mu} F_{\mu \alpha}+m^{2} F_{\alpha \beta}=0,
$$

$\left(A_{\nu}=-\frac{1}{m^{2}} \partial^{\alpha} F_{\alpha \nu}\right.$ should be substituted in $F_{\mu \nu}=$ $\partial_{\mu} A_{\nu}-\partial_{\nu} A_{\mu}$, and multiplied by $\left.m^{2}\right)$.

We also noted that the massless limit of this theory contains the Maxwell theory as a particular case. $\operatorname{In}^{16,17,22}$ we showed that it is possible to define various massless limits for the Proca-Duffin-Kemmer theory. Another one is the Ogievetskiu-Polubarinov notoph, see above. The transverse components of the AST field can be removed from the corresponding Lagrangian by means of the "new gauge transformation" $A_{\mu \nu} \rightarrow A_{\mu \nu}+\partial_{\mu} \Lambda_{\nu}-\partial_{\nu} \Lambda_{\mu}$, with the vector gauge function $\Lambda_{\mu}$.

Bargmann and Wigner claimed explicitly that they constructed $(2 s+1)$ states (the WeinbergTucker-Hammer theory has essentially $2(2 s+1)$ components). Therefore, we now apply

$$
\begin{gathered}
\Psi_{\{\alpha \beta\}}=\left(\gamma^{\mu} R\right)_{\alpha \beta}\left(c_{a} m A_{\mu}+c_{f} F_{\mu}\right)+ \\
+\left(\sigma^{\mu \nu} R\right)_{\alpha \rho}\left(c_{A} m\left(\gamma^{5}\right)_{\rho \beta} A_{\mu \nu}+c_{F} I_{\rho \beta} F_{\mu \nu}\right) .
\end{gathered}
$$

Thus, $A_{\mu}, A_{\mu \nu}$ and $F_{\mu}, F_{\mu \nu}$ have different mass dimension. The constants $c_{i}$ are some numerical dimensionless coefficients. The $\gamma^{\mu} R, \sigma^{\mu \nu} R$ and $\gamma^{5} \sigma^{\mu \nu} R$ are the symmetrical matrices. The substitution of the above expansion into the Bargmann-Wigner set, Ref., ${ }^{19}$ gives us the new Proca-like equations:

$$
\begin{aligned}
& c_{a} m\left(\partial_{\mu} A_{\nu}-\partial_{\nu} A_{\mu}\right)+c_{f}\left(\partial_{\mu} F_{\nu}-\partial_{\nu} F_{\mu}\right)= \\
& =i c_{A} m^{2} \epsilon_{\alpha \beta \mu \nu} A^{\alpha \beta}+2 m c_{F} F_{\mu \nu} \\
& c_{a} m^{2} A_{\mu}+c_{f} m F_{\mu}=i c_{A} m \epsilon_{\mu \nu \alpha \beta} \partial^{\nu} A^{\alpha \beta}+2 c_{F} \partial^{\nu} F_{\mu \nu}
\end{aligned}
$$

In the case $c_{a}=1, c_{F}=\frac{1}{2}$ and $c_{f}=c_{A}=0$ they are reduced to the ordinary Proca equations. In the general case we obtain dynamical equations which connect the photon, the notoph and their potentials. The divergent (in $m \rightarrow 0$ ) parts of field functions and those of dynamical variables should be removed by the corresponding gauge (or the Kalb-Ramond gauge) transformations. It is known that the notoph massless field is considered to be the pure longitudinal field $(h=0)$ after one takes into account $\partial_{\mu} A^{\mu \nu}=0$. Apart from these dynamical equations we can obtain a number of constraints by means of subtraction of the equations of the BargmannWigner system (instead of addition as for $(53,54)$ ). 
In fact, they give $\widetilde{F}^{\mu \nu} \sim i m A^{\mu \nu}$ and $F^{\mu} \sim m A^{\mu}$, as in Ref. ${ }^{8}$. Thus, after the suitable choice of the dimensionless coefficients $c_{i}$ the Lagrangian density for the photon-notoph field can be proposed:

$$
\begin{aligned}
\mathcal{L} & =\mathcal{L}^{\text {Proca }}+\mathcal{L}^{\text {Notoph }}=-\frac{1}{8} F_{\mu} F^{\mu}-\frac{1}{4} F_{\mu \nu} F^{\mu \nu}+ \\
& +\frac{m^{2}}{2} A_{\mu} A^{\mu}+\frac{m^{2}}{4} A_{\mu \nu} A^{\mu \nu},
\end{aligned}
$$

The limit $m \rightarrow 0$ may be taken for dynamical variables, in the end of calculations only.

Furthermore, it is logical to introduce the normalization scalar field $\varphi(x)$ and to consider the expansion:

$$
\Psi_{\{\alpha \beta\}}=\left(\gamma^{\mu} R\right)_{\alpha \beta}\left(\varphi A_{\mu}\right)+\left(\sigma^{\mu \nu} R\right)_{\alpha \beta} F_{\mu \nu} .
$$

Then, we arrive at the following set

$$
\begin{aligned}
& 2 m F_{\mu \nu}=\varphi\left(\partial_{\mu} A_{\nu}-\partial_{\nu} A_{\mu}\right)+\left(\partial_{\mu} \varphi\right) A_{\nu}-\left(\partial_{\nu} \varphi\right) A_{\mu}, \\
& \partial^{\nu} F_{\mu \nu}=\frac{m}{2}\left(\varphi A_{\mu}\right),
\end{aligned}
$$

which in the case of the constant scalar field $\varphi=$ $2 m$ can again be reduced to the system of the Proca equations. The additional constraints are

$$
\begin{aligned}
& \left(\partial^{\mu} \varphi\right) A_{\mu}+\varphi\left(\partial^{\mu} A_{\mu}\right)=0, \\
& \partial_{\mu} \widetilde{F}^{\mu \nu}=0 .
\end{aligned}
$$

At this moment, it is not yet obvious, how can we account for other equations in the $(1,0) \oplus(0,1)$ representation rigorously. One can wish to seek the generalization of the Proca system on the basis of the introduction of two mass parameters $m_{1}$ and $m_{2}$. Another equation in the $(1 / 2,0) \oplus(0,1 / 2)$ representation was discussed in Ref. ${ }^{25}$ :

$$
\left[i \gamma^{\mu} \partial_{\mu}-m_{1}-\gamma^{5} m_{2}\right] \Psi(x)=0 .
$$

The Bargmann-Wigner procedure for this system of the equations (which include the $\gamma^{5}$ matrix in the mass term) gives:

$$
\begin{aligned}
& 2 m_{1} F^{\mu \nu}+2 i m_{2} \widetilde{F}^{\mu \nu}=\varphi\left(\partial^{\mu} A^{\nu}-\partial^{\nu} A^{\mu}\right)+ \\
+ & \left(\partial^{\mu} \varphi\right) A^{\nu}-\left(\partial^{\nu} \varphi\right) A^{\mu} \\
& \partial^{\nu} F_{\mu \nu}=\frac{m_{1}}{2}\left(\varphi A_{\mu}\right),
\end{aligned}
$$

with the constraints

$$
\begin{aligned}
& \left(\partial^{\mu} \varphi\right) A_{\mu}+\varphi\left(\partial^{\mu} A_{\mu}\right)=0, \\
& \partial^{\nu} \widetilde{F}_{\mu \nu}=\frac{i m_{2}}{2}\left(\varphi A_{\mu}\right) .
\end{aligned}
$$

Next, the Tam-Happer experiments ${ }^{26}$ on two laser beams interaction did not find satisfactory explanation in the framework of the ordinary QED. On the other hand, in Ref. ${ }^{27}$ a very interesting model has been proposed. It is based on gauging the Dirac field on using the coordinate-dependent parameters $\alpha_{\mu \nu}(x)$ in

$$
\psi(x) \rightarrow \psi^{\prime}\left(x^{\prime}\right)=\Omega \psi(x), \Omega=\exp \left[\frac{i}{2} \sigma^{\mu \nu} \alpha_{\mu \nu}(x)\right] .
$$

Thus, the second "photon" was introduced. The compensating 24-component field $B_{\mu, \nu \lambda}$ reduces to the 4-vector field as follows:

$$
B_{\mu, \nu \lambda}=\frac{1}{4} \epsilon_{\mu \nu \lambda \sigma} a^{\sigma}(x) .
$$

As readily seen after comparison of these formulas with those of Refs. ${ }^{8-10}$, the second photon is nothing more than the Ogievetskiu-Polubarinov notoph within the normalization.

\section{The Bargmann-Wigner Formalism for Spin 2.}

In this Section we use the commonly-accepted procedure for the derivation of higher-spin equations. ${ }^{18}$ We begin with the equations for the 4-rank symmetric spinor:

$$
\begin{aligned}
& {\left[i \gamma^{\mu} \partial_{\mu}-m\right]_{\alpha \alpha^{\prime}} \Psi_{\alpha^{\prime} \beta \gamma \delta}=0,} \\
& {\left[i \gamma^{\mu} \partial_{\mu}-m\right]_{\beta \beta^{\prime}} \Psi_{\alpha \beta^{\prime} \gamma \delta}=0,} \\
& {\left[i \gamma^{\mu} \partial_{\mu}-m\right]_{\gamma \gamma^{\prime}} \Psi_{\alpha \beta \gamma^{\prime} \delta}=0,} \\
& {\left[i \gamma^{\mu} \partial_{\mu}-m\right]_{\delta \delta^{\prime}} \Psi_{\alpha \beta \gamma \delta^{\prime}}=0 .}
\end{aligned}
$$

We proceed expanding the field function in the complete set of symmetric matrices. In the beginning let us use the first two indices:

$$
\Psi_{\{\alpha \beta\} \gamma \delta}=\left(\gamma_{\mu} R\right)_{\alpha \beta} \Psi_{\gamma \delta}^{\mu}+\left(\sigma_{\mu \nu} R\right)_{\alpha \beta} \Psi_{\gamma \delta}^{\mu \nu} .
$$

We would like to write the corresponding equations for functions $\Psi_{\gamma \delta}^{\mu}$ and $\Psi_{\gamma \delta}^{\mu \nu}$ in the form:

$$
\begin{aligned}
& \frac{2}{m} \partial_{\mu} \Psi_{\gamma \delta}^{\mu \nu}=-\Psi_{\gamma \delta}^{\nu}, \\
& \Psi_{\gamma \delta}^{\mu \nu}=\frac{1}{2 m}\left[\partial^{\mu} \Psi_{\gamma \delta}^{\nu}-\partial^{\nu} \Psi_{\gamma \delta}^{\mu}\right] .
\end{aligned}
$$

The constraints $(1 / m) \partial_{\mu} \Psi_{\gamma \delta}^{\mu}=0 \quad$ and $(1 / m) \epsilon_{\alpha \beta}^{\mu \nu} \partial_{\mu} \Psi_{\gamma \delta}^{\alpha \beta}=0$ can be regarded as a consequence of Eqs. $(73,74)$. Next, we present the vectorspinor and tensor-spinor functions as

$$
\begin{aligned}
& \Psi_{\{\gamma \delta\}}^{\mu}=\left(\gamma^{\kappa} R\right)_{\gamma \delta} G_{\kappa}^{\mu}+\left(\sigma^{\kappa \tau} R\right)_{\gamma \delta} F_{\kappa \tau}^{\mu}, \\
& \Psi_{\{\gamma \delta\}}^{\mu \nu}=\left(\gamma^{\kappa} R\right)_{\gamma \delta} T_{\kappa}^{\mu \nu}+\left(\sigma^{\kappa \tau} R\right)_{\gamma \delta} R_{\kappa \tau}^{\mu \nu},
\end{aligned}
$$


i. e., using the symmetric matrices in indices $\gamma$ and $\delta$. Hence, the resulting tensor equations are (cf. Ref. ${ }^{28}$ )

$$
\begin{aligned}
& \frac{2}{m} \partial_{\mu} T_{\kappa}^{\mu \nu}=-G_{\kappa}^{\nu}, \\
& \frac{2}{m} \partial_{\mu} R_{\kappa \tau}^{\mu \nu}=-F_{\kappa \tau}^{\nu}, \\
& T_{\kappa}^{\mu \nu}=\frac{1}{2 m}\left[\partial^{\mu} G_{\kappa}^{\nu}-\partial^{\nu} G_{\kappa}^{\mu}\right], \\
& R_{\kappa \tau}^{\mu \nu}=\frac{1}{2 m}\left[\partial^{\mu} F_{\kappa \tau}^{\nu}-\partial^{\nu} F_{\kappa \tau}^{\mu}\right] .
\end{aligned}
$$

The constraints are re-written to

$$
\begin{aligned}
& \frac{1}{m} \partial_{\mu} G_{\kappa}^{\mu}=0, \frac{1}{m} \partial_{\mu} F_{\kappa \tau}^{\mu}=0, \\
& \frac{1}{m} \epsilon_{\alpha \beta \nu \mu} \partial^{\alpha} T_{\kappa}^{\beta \nu}=0, \frac{1}{m} \epsilon_{\alpha \beta \nu \mu} \partial^{\alpha} R_{\kappa \tau}^{\beta \nu}=0 .
\end{aligned}
$$

However, we need to make symmetrization over these two sets of indices $\{\alpha \beta\}$ and $\{\gamma \delta\}$. The total symmetry can be ensured if one contracts the function $\Psi_{\{\alpha \beta\}\{\gamma \delta\}}$ with the antisymmetric matrices $R_{\beta \gamma}^{-1},\left(R^{-1} \gamma^{5}\right)_{\beta \gamma}$ and $\left(R^{-1} \gamma^{5} \gamma^{\lambda}\right)_{\beta \gamma}$, and equate all these contractions to zero (similar to the $s=3 / 2$ case considered in Ref. [19, p. 44]). We obtain additional constraints on the tensor field functions. We explicitly showed that all field functions become to be equal to zero. Such a situation cannot be considered as a satisfactory one, because it does not give us any physical information.

We shall modify the formalism in the spirit of Ref. ${ }^{17}$ The field function (72) is now presented as

$$
\begin{aligned}
& \Psi_{\{\alpha \beta\} \gamma \delta}=\alpha_{1}\left(\gamma_{\mu} R\right)_{\alpha \beta} \Psi_{\gamma \delta}^{\mu}+\alpha_{2}\left(\sigma_{\mu \nu} R\right)_{\alpha \beta} \Psi_{\gamma \delta}^{\mu \nu}+ \\
& +\alpha_{3}\left(\gamma^{5} \sigma_{\mu \nu} R\right)_{\alpha \beta} \widetilde{\Psi}_{\gamma \delta}^{\mu \nu}
\end{aligned}
$$

with

$$
\begin{aligned}
& \Psi_{\{\gamma \delta\}}^{\mu}=\beta_{1}\left(\gamma^{\kappa} R\right)_{\gamma \delta} G_{\kappa}^{\mu}+\beta_{2}\left(\sigma^{\kappa \tau} R\right)_{\gamma \delta} F_{\kappa \tau}^{\mu}+ \\
+ & \beta_{3}\left(\gamma^{5} \sigma^{\kappa \tau} R\right)_{\gamma \delta} \widetilde{F}_{\kappa \tau}^{\mu}, \\
& \Psi_{\{\gamma \delta\}}^{\mu \nu}=\beta_{4}\left(\gamma^{\kappa} R\right)_{\gamma \delta} T_{\kappa}^{\mu \nu}+\beta_{5}\left(\sigma^{\kappa \tau} R\right)_{\gamma \delta} R_{\kappa \tau}^{\mu \nu}+ \\
+ & \beta_{6}\left(\gamma^{5} \sigma^{\kappa \tau} R\right)_{\gamma \delta} \widetilde{R}_{\kappa \tau}^{\mu \nu}, \\
& \widetilde{\Psi}_{\{\gamma \delta\}}^{\mu \nu}=\beta_{7}\left(\gamma^{\kappa} R\right)_{\gamma \delta} \widetilde{T}_{\kappa}^{\mu \nu}+\beta_{8}\left(\sigma^{\kappa \tau} R\right)_{\gamma \delta} \widetilde{D}_{\kappa \tau}^{\mu \nu}+ \\
+ & \beta_{9}\left(\gamma^{5} \sigma^{\kappa \tau} R\right)_{\gamma \delta} D_{\kappa \tau}^{\mu \nu} .
\end{aligned}
$$

Hence, the function $\Psi_{\{\alpha \beta\}\{\gamma \delta\}}$ can be expressed as a sum of nine terms. The corresponding dynamical equations are given in the following form:

$$
\begin{aligned}
& \frac{2 \alpha_{2} \beta_{4}}{m} \partial_{\nu} T_{\kappa}^{\mu \nu}+\frac{i \alpha_{3} \beta_{7}}{m} \epsilon^{\mu \nu \alpha \beta} \partial_{\nu} \widetilde{T}_{\kappa, \alpha \beta}=\alpha_{1} \beta_{1} G_{\kappa}^{\mu}(87) \\
& \frac{2 \alpha_{2} \beta_{5}}{m} \partial_{\nu} R_{\kappa \tau}^{\mu \nu}+\frac{i \alpha_{2} \beta_{6}}{m} \epsilon_{\alpha \beta \kappa \tau} \partial_{\nu} \widetilde{R}^{\alpha \beta, \mu \nu}+ \\
& +\frac{i \alpha_{3} \beta_{8}}{m} \epsilon^{\mu \nu \alpha \beta} \partial_{\nu} \widetilde{D}_{\kappa \tau, \alpha \beta}-\frac{\alpha_{3} \beta_{9}}{2} \epsilon^{\mu \nu \alpha \beta} \epsilon_{\lambda \delta \kappa \tau} D_{\alpha \beta}^{\lambda \delta}= \\
& =\alpha_{1} \beta_{2} F_{\kappa \tau}^{\mu}+\frac{i \alpha_{1} \beta_{3}}{2} \epsilon_{\alpha \beta \kappa \tau} \widetilde{F}^{\alpha \beta, \mu} \\
& 2 \alpha_{2} \beta_{4} T_{\kappa}^{\mu \nu}+i \alpha_{3} \beta_{7} \epsilon^{\alpha \beta \mu \nu} \widetilde{T}_{\kappa, \alpha \beta}= \\
& =\frac{\alpha_{1} \beta_{1}}{m}\left(\partial^{\mu} G_{\kappa}^{\nu}-\partial^{\nu} G_{\kappa}^{\mu}\right), \\
& 2 \alpha_{2} \beta_{5} R_{\kappa \tau}^{\mu \nu}+i \alpha_{3} \beta_{8} \epsilon^{\alpha \beta \mu \nu} \widetilde{D}_{\kappa \tau, \alpha \beta}+ \\
& +i \alpha_{2} \beta_{6} \epsilon_{\alpha \beta \kappa \tau} \widetilde{R}^{\alpha \beta, \mu \nu}-\frac{\alpha_{3} \beta_{9}}{2} \epsilon^{\alpha \beta \mu \nu} \epsilon_{\lambda \delta \kappa \tau} D_{\alpha \beta}^{\lambda \delta}= \\
& =\frac{\alpha_{1} \beta_{2}}{m}\left(\partial^{\mu} F_{\kappa \tau}^{\nu}-\partial^{\nu} F_{\kappa \tau}^{\mu}\right)+ \\
& +\frac{i \alpha_{1} \beta_{3}}{2 m} \epsilon_{\alpha \beta \kappa \tau}\left(\partial^{\mu} \widetilde{F}^{\alpha \beta, \nu}-\partial^{\nu} \widetilde{F}^{\alpha \beta, \mu}\right) .
\end{aligned}
$$

In general, the coefficients $\alpha_{i}$ and $\beta_{i}$ may now carry some dimension. The essential constraints can be found in Ref. ${ }^{29}$ They are the results of contractions of the field function with six antisymmetric matrices, as above. As a discussion, we note that in such a framework we have physical content because only certain combinations of field functions can be equal to zero. In general, the fields $F_{\kappa \tau}^{\mu}, \widetilde{F}_{\kappa \tau}^{\mu}, T_{\kappa}^{\mu \nu}, \widetilde{T}_{\kappa}^{\mu \nu}$, and $R_{\kappa \tau}^{\mu \nu}, \widetilde{R}_{\kappa \tau}^{\mu \nu}, D_{\kappa \tau}^{\mu \nu}, \widetilde{D}_{\kappa \tau}^{\mu \nu}$ correspond to different physical states. The equations describe couplings one state to another.

Furthermore, from the set of equations (87-90) one obtains the second-order equation for symmetric traceless tensor of the second rank $\left(\alpha_{1} \neq 0, \beta_{1} \neq 0\right)$ :

$$
\frac{1}{m^{2}}\left[\partial_{\nu} \partial^{\mu} G_{\kappa}^{\nu}-\partial_{\nu} \partial^{\nu} G_{\kappa}^{\mu}\right]=G_{\kappa}^{\mu}
$$

After the contraction in indices $\kappa$ and $\mu$ this equation is reduced to:

$$
\begin{aligned}
& \partial_{\mu} G_{\kappa}^{\mu}=F_{\kappa} \\
& \frac{1}{m^{2}} \partial_{\kappa} F^{\kappa}=0,
\end{aligned}
$$

i. e., the equations which connect the analogue of the energy-momentum tensor and the analogue of the 4 -vector potential. See also the works on the notivarg concept. ${ }^{30}$ Further investigations may provide additional foundations to "surprising" similarities of gravitational and electromagnetic equations in the low-velocity limit, Refs. ${ }^{31-34}$ 


\section{Interactions with Fermions.}

The possibility of terms such as $\sigma \cdot\left[\mathbf{A} \times \mathbf{A}^{*}\right]$ appears to be related to the matters of chiral interactions. ${ }^{35,36}$ As we are now convinced, the Dirac field operator can be always presented as a superposition of the self- and anti-self charge conjugate field operators (cf. Ref. ${ }^{38}$ ). The anti-self charge conjugate part gives the self charge conjugate part after multiplying by the $\gamma^{5}$ matrix, and vice versa. We derived

$$
\begin{aligned}
& {\left[i \gamma^{\mu} D_{\mu}^{*}-m\right] \psi_{1}^{s}=0,} \\
& {\left[i \gamma^{\mu} D_{\mu}-m\right] \psi_{2}^{a}=0 .}
\end{aligned}
$$

Both equations lead to the interaction terms such as $\sigma \cdot\left[\mathbf{A} \times \mathbf{A}^{*}\right]$ provided that the 4 -vector potential is considered as a complex function(al). In fact, from (94) we have:

$$
i \sigma^{\mu} \nabla_{\mu} \chi_{1}-m \phi_{1}=0, i \tilde{\sigma}^{\mu} \nabla_{\mu}^{*} \phi_{1}-m \chi_{1}=0 .
$$

And, from (95) we have

$$
i \sigma^{\mu} \nabla_{\mu}^{*} \chi_{2}-m \phi_{2}=0, i \tilde{\sigma}^{\mu} \nabla_{\mu} \phi_{2}-m \chi_{2}=0 .
$$

The meanings of $\sigma^{\mu}$ and $\tilde{\sigma}^{\mu}$ are obvious from the definition of $\gamma$ matrices. The derivatives are defined:

$$
D_{\mu}=\partial_{\mu}-i e \gamma^{5} C_{\mu}+e B_{\mu}, \nabla_{\mu}=\partial_{\mu}-i e A_{\mu},
$$

and $A_{\mu}=C_{\mu}+i B_{\mu}$. Thus, relations with the magnetic monopoles can be established.

From the above systems we extract the terms $\pm e^{2} \sigma^{i} \sigma^{j} A_{i} A_{j}^{*}$, which lead to the discussed terms. ${ }^{35,36}$ We would like to note that the terms of the type $\boldsymbol{\sigma} \cdot\left[\mathbf{A} \times \mathbf{A}^{*}\right]$ can be reduced to $(\boldsymbol{\sigma} \cdot \nabla) V$, where $V$ is the scalar potential.

Furthermore, one can come to the same conclusions not applying to the constraints on the creation/annihilation operators (which we previously choose for clarity and simplicity $\left.\mathrm{in}^{36}\right)$. It is possible to work with self/anti-self charge conjugate fields and the Majorana anzatzen. Thus, it is the $\gamma^{5}$ transformation which distinguishes various field configurations (helicity, self/anti-self charge conjugate properties etc) in the coordinate representation in the considered cases.

\section{Boson Interactions.}

The most general relativistic-invariant Lagrangian for the symmetric 2nd-rank tensor is

$$
\begin{gathered}
\mathcal{L}=-\alpha_{1}\left(\partial^{\alpha} G_{\alpha \lambda}\right)\left(\partial_{\beta} G^{\beta \lambda}\right)-\alpha_{2}\left(\partial_{\alpha} G^{\beta \lambda}\right)\left(\partial^{\alpha} G_{\beta \lambda}\right) \\
-\alpha_{3}\left(\partial^{\alpha} G^{\beta \lambda}\right)\left(\partial_{\beta} G_{\alpha \lambda}\right)+m^{2} G_{\alpha \beta} G^{\alpha \beta} .
\end{gathered}
$$

It leads to the equation

$$
\left[\alpha_{2}\left(\partial_{\alpha} \partial^{\alpha}\right)+m^{2}\right] G^{\{\mu \nu\}}+\left(\alpha_{1}+\alpha_{3}\right) \partial^{\{\mu \mid}\left(\partial_{\alpha} G^{\alpha \mid \nu\}}\right)=0 .
$$

In the case $\alpha_{2}=1>0$ and $\alpha_{1}+\alpha_{3}=-1$ it coincides with Eq. (91). There is no any problem to obtain the dynamical invariants for the fields of the spin 2 from the above Lagrangian. The mass dimension of $G^{\mu \nu}$ is [energy $]^{1}$. We now present possible relativistic interactions of the symemtric 2nd-rank tensor. The simplest ones should be the the following ones:

$$
\begin{aligned}
\mathcal{L}_{(1)}^{i n t} & \sim G_{\mu \nu} F^{\mu} F^{\nu}, \\
\mathcal{L}_{(2)}^{i n t} & \sim\left(\partial^{\mu} G_{\mu \nu}\right) F^{\nu}, \\
\mathcal{L}_{(3)}^{i n t} & \sim G_{\mu \nu}\left(\partial^{\mu} F^{\nu}\right) .
\end{aligned}
$$

The term $\left(\partial_{\mu} G_{\alpha}^{\alpha}\right) F^{\mu}$ vanishes due to the constraint of tracelessness. The interaction with the notoph can be related o the scalar-tensor theories of gravity.

It is also interesting to note that thanks to the possible terms

$$
V(F)=\lambda_{1}\left(F_{\mu} F^{\mu}\right)+\lambda_{2}\left(F_{\mu} F^{\mu}\right)\left(F_{\nu} F^{\nu}\right)
$$

we can give the mass to the $G_{00}$ component of the spin-2 field. This is due to the possibility of the Higgs spontaneous symmetry breaking: ${ }^{37}$

$$
F^{\mu}(x)=\left(\begin{array}{c}
v+\partial_{0} \chi(x) \\
g^{1} \\
g^{2} \\
g^{3}
\end{array}\right)
$$

with $v$ being the vacuum expectation value, $v^{2}=$ $\left(F_{\mu} F^{\mu}\right)=-\lambda_{1} / 2 \lambda_{2}>0$. Other degrees of freedom of the 4 -vector field are removed since they are the Goldstone bosons. It was stated that "for any continuous symmetry which does not preserve the ground state, there is a massless degree of freedom which decouples at low energies. This mode is called the Goldstone (or Nambu-Goldstone) particle for the symmetry". As usual, the Goldstone modes should be important in giving masses to the three vector bosons. As one can easily seen, this expression does not permit an arbitrary phase for $F^{\mu}$, which is only possible if the 4 -vector would be the complex one.

Next, since the interaction of fermions with notoph, for instance, are that of the order $\sim e^{2}$ since the beginning (as opposed to the fermion current interaction with the 4 -vector potential $A_{\mu}$ ) in the Lagrangian, it is more difficult to observe it. However, as far as I know, the theoretical precision calculus 
in QED (the Landé factor, the anomalous magnetic moment, the hyperfine splittings in positronium and muonium, and the decay rate of $o-P s$ and $p-P s$ ) are near the order corresponding to the 4th-5th loops, where the difference may appear with the experiments, cf. 39,40

\section{Conclusions}

We considered the Bargmann-Wigner formalism in order to derive the equations for the AST fields, and for the symmetric tensor of the 2 nd rank. We introduced the additional normalization scalar field in the Bargmann-Wigner formalism in order to account for possible physical significance of the OgievetskiiPolubarinov-Kalb-Ramond modes. Both the antisymmetric tensor fields and the 4-vector fields may have third helicity state in the massless limit from a theoretical viewpoint. This problem is connected with the problem of the observability of the gauge. ${ }^{21}$ We introduced the additional symmetric matrix in the Bargmann-Wigner expansion $\left(\gamma^{5} \sigma^{\mu \nu} R\right)$ in order to account for the dual fields. The consideration was similar to Ref. ${ }^{41}$ The problem was discussed, what are the the correct definitions of the energy-momentum tensor and other Nöther currents in the electromagnetic theory, the relativistic theory of gravitation, the general relativity, and their generalizations. Furthermore, we discussed the interactions of notoph, photon and graviton. Probably, the notivarg should also be taken into account. In order to analize its dynamical invariants and interactions one should construct Lagrangian from the analogs of the Riemann tensor, such as $\widetilde{D}^{\mu \nu, \alpha \beta}$. For instance, the interaction notoph-graviton may give the mass to spin-2 particles in the way similar to the spontaneous-symmetry-breaking Higgs formalism.

\section{Acknowledgments}

I am grateful to the referee of "International Journal of Modern Physics" and "Foundation of Physics", whose advice of the mass dimension (normalization) of the fields was very useful. I acknowledge discussions with participants of recent conferences on Symmetries.

\section{References}

1. D. V. Ahluwalia and D. J. Ernst, Int. J. Mod. Phys. E2 (1993) 397.
2. D. V. Ahluwalia and M. Sawicki, Phys. Rev. D47 (1993) 5161.

3. V. V. Dvoeglazov, Int. J. Theor. Phys. 37 (1998), 1915; hep-th/9611068.

4. V. V. Dvoeglazov, Hadronic J. Suppl. 12 (1997) 221.

5. S. Weinberg, Phys. Rev. B133 (1964) 1318; ibid. B134 (1964) 882; ibid. 181 (1969) 1893.

6. R. H. Tucker and C. L. Hammer, Phys. Rev. D3 (1971) 2448.

7. E. Durandin and A. Erschow, Phys. Z. Sowjet. 12 (1937) 466; F. Belinfante, Physica 6 (1939) 887.

8. V. I. Ogievetskiŭ and I. V. Polubarinov, Yadern. Fiz. 4 (1966) 216 [English translation: Sov. J. Nucl. Phys. 4 (1967) 156].

9. K. Hayashi, Phys. Lett. B44 (1973) 497.

10. M. Kalb and P. Ramond, Phys. Rev. D9 (1974) 2273.

11. L. V. Avdeev and M. V. Chizhov, Phys. Lett. B321 (1994) 212; hep-th/9407067; M. V. Chizhov, Phys. Part. Nucl. 42 (2011) 93.

12. N. N. Bogoliubov and D. V. Shirkov, Introduction to the Theory of Quantized Fields. (Moscow, Nauka, 1973).

13. Yu. V. Novozhilov, Introduction to Elementary Particle Theory. (Pergamon Press, Oxford, 1975).

14. C. Itzykson and J.-B. Zuber, Quantum Field Theory. (McGraw-Hill Book Co. New York, 1980).

15. S. Weinberg, The Quantum Theory of Fields. Vol. I. Foundations. (Cambridge University Press, 1995), Chapter 5.

16. V. V. Dvoeglazov, Czech. J. Phys. 50 (2000) 225; , hep-th/9712036.

17. V. V. Dvoeglazov, Phys. Scripta 64 (2001) 201, physics/9804010.

18. V. Bargmann and E. P. Wigner, Proc. Natl. Acad. Sci. (USA) 34 (1948) 211.

19. D. Luriè, Particles and Fields (Interscience Publishers, 1968). Chapter 1.

20. M. Kirchbach, Mod. Phys. Lett. A12 (1997) 2373.

21. A. Staruszkiewicz, Acta Phys. Polon. B13 (1982) 617; ibid. 14 (1983) 63, 67, 903; ibid. 15 (1984) 225; ibid 23 (1992) 591.

22. V. V. Dvoeglazov, Electromagnetic Phenomena 1, No. 4 (1998) 465; hep-th/9604148.

23. H. C. Ohanian, Am. J. Phys. 54 (1986) 500.

24. Yu. M. Shirokov, ZhETF 33 (1957) 861, ibid. 1196 [English translation: Sov. Phys. JETP 6 (1958) 664, ibid. 919]; Chou Kuang-chao and M. I. Shirokov, ZhETF 34 (1958) 1230 [English translation: Sov. Phys. JETP 7 (1958) 851].

25. N. D. Sen Gupta, Nucl. Phys. B4 (1967) 147; A. Raspini, Fizika B5 (1996) 159.

26. A. C. Tam and W. Happer, Phys. Rev. Lett. 38 (1977) 278.

27. P. C. Naik and T. Pradhan, J. Phys. A14 (1981) 2795; T. Pradhan, R. P. Malik and P. C. Naik, Pramana J. Phys. 24 (1985) 77.

28. G. Marques and D. Spehler, Mod. Phys. Lett. A13 (1998) 553. 
29. V. V. Dvoeglazov, Adv. Appl. Clifford Algebras 10 (2000) 7, math-ph/9805017.

30. S. Deser, Nucl. Phys. B184 (1981) 333; J. Rembieliński and W. Tybor, Acta Phys. Polon. B22 (1991) 439; ibid. 447; M. Bakalarska and W. Tybor, On Notivarg Propagator. Preprint hep-th/9801065; On the Deser-Siegel-Townsend Notivarg. Preprint hep-ph/9801216, Łódź, Poland.

31. S. Weinberg, Gravitation and Cosmology. (John Wiley \& Sons, New York, 1972).

32. A. Logunov and M. Mestvirishvili, The Relativistic Theory of Gravitation. (Mir Publishers, Moscow, 1989).

33. V. L. Ginzburg, D. A. Kirzhnitz, public discussions about the RTG ( some of their seminars have been attended by me since 1983).

34. E. A. Notte-Cuello and W. A. Rodrigues Jr., Int. J. Mod. Phys. D16 (2007) 1027, math-ph/0608017.

35. V. V. Dvoeglazov, Electromagnetic Phenomena, 2, No. 1 (5) (2001) 3, hep-ph/9801287.

36. V. V. Dvoeglazov, Acta Phys. Slovaca 50 (2000) 629.

37. P. Higgs, Phys. Rev. Lett. 13 (1964) 508.

38. A. Barut and G. Ziino, Mod. Phys. Lett. A8 (1993) 1099; G. Ziino, Int. J. Mod. Phys. A11 (1996) 2081.

39. V. V. Dvoeglazov, R. N. Faustov and Yu. N. Tyukhtyaev, Phys. Part. Nucl. 25 (1994) 58.

40. T. Kinoshita, http://www.riken.jp/labwww/theory/colloquium/kinoshita.pdf .

41. V. V. Dvoeglazov, Int. J. Mod. Phys. CS 3 (2011) 121. 